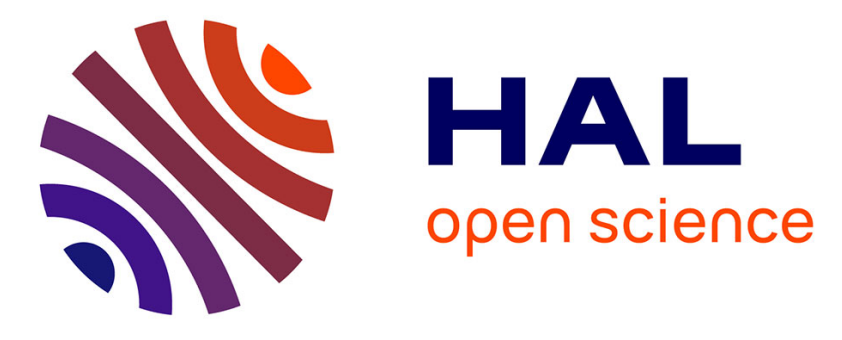

\title{
A Tumor-mimic Model for Evaluating the Accuracy of HIFU Preclinical Studies: An In Vivo Study.
}

William Apoutou N'Djin, David Melodelima, Hubert Parmentier, Michel Rivoire, Jean Yves Chapelon

\section{- To cite this version:}

William Apoutou N'Djin, David Melodelima, Hubert Parmentier, Michel Rivoire, Jean Yves Chapelon. A Tumor-mimic Model for Evaluating the Accuracy of HIFU Preclinical Studies: An In Vivo Study.. Conference proceedings: .. Annual International Conference of the IEEE Engineering in Medicine and Biology Society. IEEE Engineering in Medicine and Biology Society. Annual Conference, 2007, 1, pp.3544-3547. 10.1109/IEMBS.2007.4353096 . inserm-00192601

\section{HAL Id: inserm-00192601 https://www.hal.inserm.fr/inserm-00192601}

Submitted on 28 Nov 2007

HAL is a multi-disciplinary open access archive for the deposit and dissemination of scientific research documents, whether they are published or not. The documents may come from teaching and research institutions in France or abroad, or from public or private research centers.
L'archive ouverte pluridisciplinaire HAL, est destinée au dépôt et à la diffusion de documents scientifiques de niveau recherche, publiés ou non, émanant des établissements d'enseignement et de recherche français ou étrangers, des laboratoires publics ou privés. 


\title{
A Tumor-mimic Model for Evaluating the Accuracy of HIFU Preclinical Studies: An In Vivo Study
}

\author{
W. A. N'Djin, D. Melodelima, H. Parmentier, M. Rivoire and J. Y. Chapelon
}

\begin{abstract}
To date, the efficacy of ablative technologies such as HIFU for the treatment of liver tumors in humans has been studied in animal models without tumors or in small animals like rats and rabbits with established tumors. Because of the small size of these animals, the lesion produced by HIFU devices has to be small. Thus, the local and systemic effects of the treatment as encountered in humans cannot be studied. The purpose of this study was to use an in vivo tumor-mimic model to evaluate the accuracy of HIFU ablation in the liver in preclinical studies. Tumor mimics were created in in vivo porcine livers by injecting a 1-cc warm mixture of agarose, cellulose, glycerol and methylene blue, which formed $1-\mathrm{cm}$ hyperechoic discrete lesions on sonograms. Three studies were carried out: (i) in vitro experiments were conducted to study the acoustical proprieties of the tumor mimics; (ii) in vivo experiments were conducted in 10 pigs to evaluate the tolerance of the tumor mimics when injected in the liver; (iii) ultrasoundguided HIFU ablation was performed in 10 pigs to demonstrate that it is possible to treat a predetermined zone accurately. It was shown that the acoustical properties of tumor mimics are visible in sonograms and do not modify the shape and dimensions of HIFU lesions. The local and biological tolerance of tumor mimics was excellent. In addition, it was demonstrated that the average difference between the predetermined location of the HIFU ablation and the actual coagulated area was $32 \%$. Therefore, this tumor mimic can be used to teach HIFU ablation before starting clinical studies, especially if the ultrasound device is to be used manually, as the one presented in this study was.
\end{abstract}

\section{INTRODUCTION}

To date, two approaches have been used to evaluate the treatment of liver tumors by High-Intensity Focused Ultrasound (HIFU): (i) Testing the effects of HIFU on real tumors and the liver-tumor models available are those in small animals (rats and rabbits); (ii) Testing the effects of HIFU on pig liver because it is an ideal animal for studying HIFU treatment, in view of its size and similar physiology to

Manuscript received April 2, 2007.

This work was supported by funding from the French ACI Cancéropôle Grand Ouest 2004 "CDTU, Cancer Diagnosis and Treatment by Ultrasound" (number 2004-01).

W. A. N'Djin, D. Melodelima and H. Parmentier are with «l'Institut National de la Santé et de la Recherche Médicale, INSERM U556 », Lyon, 69424 Cedex 03, France (phone: +33-(0)4-72-68-1930; fax: +33-(0)4-7268-1931; e-mail: ndjin@lyon.inserm.fr, melodelima@lyon.inserm.fr, hubert.parmentier@chu-lyon.fr).

M. Rivoire is with «l'Institut de Chirugie Expérimentale, ICE », Lyon, 69373, France (phone: +33-(0)4-78-78-28-34; e-mail: rivoire@lyon.fnclcc.fr).

J. Y. Chapelon is with INSERM U556, Lyon, 69424 Cedex 03, France (phone: +33-(0)4-72-68-1930; fax: +33-(0)4-72-68-1931; e-mail: chapelon@lyon.inserm.fr). humans. In small animals, the size of the liver is too different of those in humans. Thus, the local and systemic effects of prolonged treatment and the effects of ablative treatment on liver tumors as encountered in humans cannot be studied. In addition, there is no established liver-tumor model in pigs and HIFU studies are generally performed on healthy animals. However, it is essential to study the accuracy of HIFU treatments on a localized zone as it was demonstrated that treatment margins all around a tumor reduce the recurrence rate [1-2]. The difficulty in developing such a tumor model in pigs led us to alternative solutions. Ideally, a tumor model visible on sonogram would be well suited to evaluating the accuracy of HIFU treatment in treating a predetermined zone in the liver with treatment margins.

In this study, we propose to use an injectable sonographic tumor mimic, as described by D.J. Scott et al. [3] in a study on radiofrequency ablation as a method for evaluating the accuracy of HIFU preclinical treatments. These tumor mimics are not expected to substitute real tumors but being used as a target, they could be excellent tools for assessing whether the position of the induced necrosis actually corresponds to the theoretical position of the HIFU ablation. To date, this model has never been studied acoustically, nor over long periods of time. Three studies were therefore conducted. First, several acoustical proprieties of tumor mimics were studied (attenuation, velocity of propagation and acoustic impedance) in order to verify whether they should induce modifications during HIFU treatments in liver tissue. Second, tumor mimics were injected in vivo in 10 pig livers and radiological, clinical, histological and biological monitoring of the pigs and the tumor mimics was performed to assess long-term (30 days) tolerance, feasibility and timeindependancy. Third, HIFU exposures were performed in vivo at a fixed distance $(10 \mathrm{~mm})$ from the tumor mimic to ensure that the ultrasound lesions were actually made at the theoretical position. Juxtaposed exposures on the tumor mimic were also performed to determine the feasibility of surrounding a targeted zone with a margin $10 \mathrm{~mm}$ wide.

\section{MATERIALS AND METHODS}

\section{A. Acoustical parameters of tumor-mimics}

This approach aims to check that the mimic model is suitable for carrying out in vivo studies in pig livers by comparing mimic-model acoustical parameters. Tumor mimics were prepared as described in [3]. A 5-MHz plane 
transducer $(8 \times 14 \mathrm{~mm})$ and a 0.4-mm needle-type hydrophone (PZT-Z44-0400, SEA) were used for transmission measurements. The acoustical parameters studied were attenuation, ultrasound propagation velocity in the tumor mimics and acoustic impedance. All measurements were made at room temperature.

The absolute pressure attenuation value of the tumor mimic and of a sample of pig liver was determined by calculating the ratio between the pressure measured with and without the material in a frequency band of 1 to $7.5 \mathrm{MHz}$ with a step of $0.5 \mathrm{MHz}$. All measurements were repeated 10 times to ensure reproducibility of the results.

The ultrasound time of flight with and without tumor mimics was measured. Measurements were performed twice at 5 different frequencies (range: $1-5 \mathrm{MHz}$ ) with a step of 1 $\mathrm{MHz}$. The acoustic impedance of tumor mimics was then calculated after measuring the mass density.

\section{B. Tolerance and time-independancy of tumor-mimics}

This study was conducted on 10 pigs which were not involved in the next studies in order to evaluate solely the tolerance and the time-independancy of the tumor-mimics. HIFU exposures were not performed in this study not to influence the tolerance. Animals with an average weight of $23.2 \pm 2.7 \mathrm{~kg}(19.6-27.3 \mathrm{~kg})$ were used. All procedures were performed in the laboratory for experimental surgery (DSV 693880501) of the Centre Léon Bérard in Lyon (F69008, France). Animal experiments were performed under an approved research protocol, in accordance with the World Medical Assembly Declaration of Helsinki. These experiments conformed to the requirements of the local office of animal experimentation and were in accordance with the legal conditions of the French National Commission on Animal Experimentation.

A $25-\mathrm{cm}$ median laparotomy was carried out from the xiphoid process. Laparotomy was actually chosen in order to be able to compare the results of this study to the next study for evaluating the accuracy of HIFU treatments. A $12-\mathrm{MHz}$ linear ultrasound imaging probe and a $7.5-\mathrm{MHz}$ sectorial ultrasound imaging probe were used during this in vivo study. Each pig received a mean of $3.9 \pm 0.3$ (range: $3-5$ ) intrahepatic injections of the warm mixture. This mixture is liquid at $65^{\circ} \mathrm{C}$ and solid at $37^{\circ} \mathrm{C}$ (liver temperature). The tumor mimic was injected, under ultrasound imaging control, into the liver using a syringe filled with viscous fluid at $65^{\circ} \mathrm{C}$ (between 0.5 and $1.5 \mathrm{~mL}$ ). Liver movements caused by breathing were eliminated by maintaining pigs under apnea during injection. The tumor mimic hardened in the liver within one minute, producing a hyperechoic discrete lesion. Injections were considered successful if a discrete, homogeneous and hyperechoic tumor mimic of approximately $1 \mathrm{~cm}$ in diameter was created. Clinical follow-up included red blood cell count, liver enzymes, urea, creatinine and creatine kinase, and clotting tests.

The animals were euthanized on D0 (2 pigs), D4 (4 pigs),
D8 (2 pigs), D15 (1 pig) and D30 (1 pig) after the injections. Euthanasia was performed under general anesthesia using an intravenous injection of $20 \mathrm{~mL}$ of potassium chloride at $10 \%$ and a complete hepatectomy was performed. Tumor mimics were removed and sectioned transversally and in the other two dimensions. Representative tumor mimics were stained using routine hematoxylin and eosin (H\&E) methods for histopathological analysis.

\section{Evaluating the accuracy of HIFU preclinical studies}

This study was performed on a new set of 10 pigs. Animals with an average weight of $33.4 \pm 2.3 \mathrm{~kg}(29.0-43.0$ $\mathrm{kg}$ ) were used and anesthetized as described previously. Thermal ablations were produced using an intraoperative HIFU probe (Fig. 1) operating at $3 \mathrm{MHz}$ with integrated ultrasound imaging probe (7.5-MHz sectorial transducer). Eventually, this device was developed for human treatments. It is for use by hand intraoperatively in order to avoid, primarily, the difficulties induced by the ribs. A sterile envelope containing circulating cooling fluid (Ablasonic $\AA$, EDAP, France) makes it possible to prevent high temperature rises in the transducers during HIFU exposures. An interface enabled the user to visualize the focal point of the HIFU device (radius of curvature of $70 \mathrm{~mm}$ ) on the ultrasound image, as well as on the tissues due to coagulate. A preliminary study determined optimal parameters for in vivo HIFU treatment in pig livers up to D30 using the HIFU device described above [4].

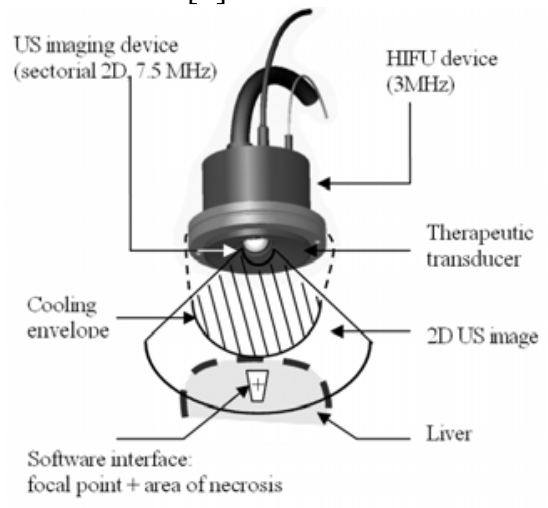

Fig. 1. Schematic diagram of the HIFU device and software interface.

A first set of HIFU exposures were performed on 5 pigs after the injection of $5.2 \pm 0.22$ (range: 5.0-6.0) tumor mimics per animal. HIFU lesions were produced $10 \mathrm{~mm}$ from the border of the tumor mimics visualized on sonograms, in order to show that thermal ablation can be produced in the liver accurately without damaging tissue that must be preserved.

Treatment margins of $10 \mathrm{~mm}$ around the tumors are typically used in ablative techniques to reduce the recurrence rate. Therefore, another set of HIFU thermal ablations were performed on 5 pigs after the injection of 2.8 \pm 0.4 (range: 2.0-4.0) tumor mimics per animal. To surround the tumor mimic with margins $10 \mathrm{~mm}$ wide, on average $9.2 \pm 0.4$ (range 9.0-12.0) juxtaposed HIFU lesions 
were performed. The probe displacements between each exposure were performed manually under ultrasound imaging control. A scan of the tumor mimic with the $2 \mathrm{D}$ ultrasound imaging probe enabled the detection of boundaries in all directions, and the focal zone of the first HIFU exposure was placed in the center of the tumor mimic.

At the end of the procedure, the liver was removed and sliced to inspect ultrasound effects visually. The theoretical distances displayed on sonograms between HIFU lesions and tumor mimics were compared to the measurements on samples. Each hepatic lesion was sectioned transversally and then sliced into samples $5 \mathrm{~mm}$ thick to determine if thermal damage was homogeneous. The dimensions of the necroses were measured using a caliper. The average difference between the predetermined location of the HIFU ablation and the actual coagulated area was quantified by calculating the percentage error between the theoretical distance (or margins) of $10 \mathrm{~mm}$ and the measurements on the samples.

\section{RESULTS}

\section{A. Acoustical parameters of tumor-mimics}

In the band frequency $1-7.5 \mathrm{MHz}$, the average attenuation of tumor mimics was non-linear and was interpolated by a power function. The correlation coefficient was 0.98 . The $\log$-log slope was 1.63 and the attenuation at $1 \mathrm{MHz}$ was 0.41 dB.cm-1(Fig. 2). In healthy liver tissue, the log-log slope was 1.00 and the attenuation at $1 \mathrm{MHz}$ was 0.68 dB.cm-1 [5].

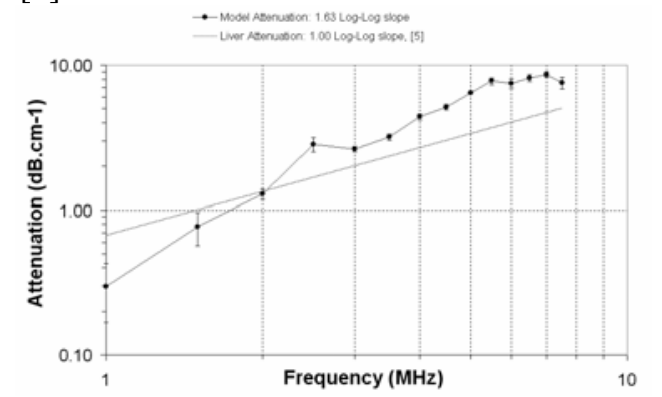

Fig. 2. Acoustical attenuation in the model between 1-7.5 MHz

A series of 10 measurements (2 measurements at 5 different frequencies) established the time of flight decrease in the model to be $0.164 \pm 0.004 \mu$ s (range: $0.160-0.180$ $\mu \mathrm{s})$. The average speed of sound in the model was measured to be $1522.6 \pm 1.0 \mathrm{~m} . \mathrm{s}-1$ (range 1522.1-1524.9 m.s-1), assuming the speed of sound in water was $1500 \mathrm{~m} . \mathrm{s}-1$. The tumor mimics' lenght $(76.2 \mathrm{~mm})$, width $(49.0 \mathrm{~mm})$ and depth $(12.8 \mathrm{~mm})$ were used to measure the acoustic impedance of the material. Its mass was $57.8 \mathrm{~g}$, giving a mass density of $1209.2 \mathrm{~kg} \cdot \mathrm{m}-3$. Consequently, the acoustic impedance in the model was $1.841 \pm 0.001$ MRay (range: 1.841-1.884 MRay).

\section{B. Tolerance and time-independancy of tumor-mimics}

Out of 44 injections, 39 (88\%) were successful. A total of 5 injections failed due to injection into a blood vessel (two cases), injection into a bile duct (one case), failure of the fluid solution to produce a discrete lesion (one case), and the failure of the injection to appear on sonograms (one case). One pig died on D0 following a vena cava hemorrhage due to excessive traction on the liver not related to the injection. In total, $80 \%$ of the tumor mimics were homogeneous and ellipsoidal (Figs. 3a, 3b and 4b).
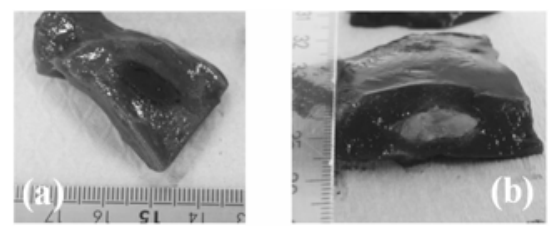

Fig. 3. Macroscopic view of tumor-mimics, (a) at day 0 , (b) at day 30

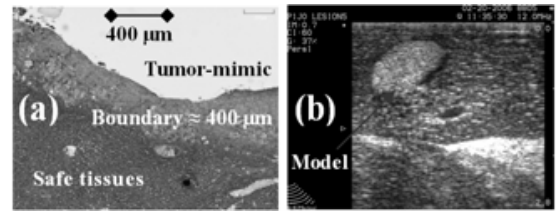

Fig. 4. (a) Histologic view of a tumor-mimic in an in vivo pig liver at day 30, (b) Hyperechoic tumor-mimic in an in vivo pig liver (12 $\mathrm{MHz}$ ultrasound imaging probe)

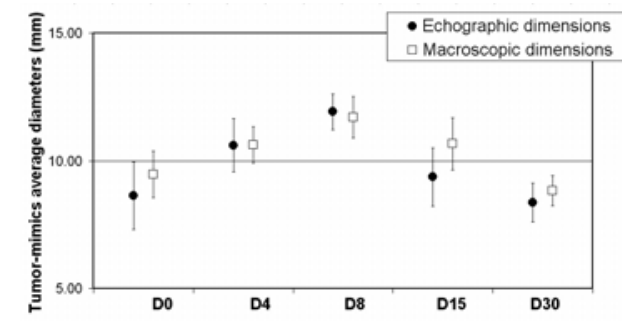

Fig. 5. Evolution of the tumor-mimic average dimensions and comparison between ultrasound and macroscopic measurements

The local and biological tolerance of the treatment was excellent. Pigs recovered from the procedures within two hours of terminating anesthesia and resumed eating and normal behavior. Blood-test results were all normal up to D30. Histological analysis revealed no changes in healthy hepatic tissue beyond a $500 \mu \mathrm{m}$ radius of the injected tumor mimic (Fig. 4a). Ultrasound imaging (Fig. 4b) and macroscopic analysis (Fig. 3) show no evidence of changes in the geometry of the tumor mimics throughout the 30 days of the study. On macroscopic samples, a discoloration was observed from D4. The dimensions of the injected tumor mimics were measured using ultrasound imaging by scanning their volumes and identifying the largest diameter. The other two diameters were then measured using orthogonal planes. On the sample, the tumors-mimics were measured following the three spacial dimensions. The average diameters on sonogram were compared to those on the samples (Fig. 5). The mean values on D0 for the largest diameters and the other two orthogonal diameters of tumor mimics for 1-cc injections were $8.6 \pm 2.5 \mathrm{~mm}$ (range: $2.6-$ $20.0 \mathrm{~mm}$ ). On D0, mean difference between ultrasound and macroscopic measurements were $8.7 \%$. 


\section{Evaluating the accuracy of HIFU preclinical studies}

The first set of HIFU exposures led to 26 HIFU lesions at a fixed distance from the tumor-mimic model (Fig. 6). The average distances recorded between the tumor mimic and the center of the lesion in this series of exposures was $11.3 \pm$ $1.0 \mathrm{~mm}$ (range: $8-14 \mathrm{~mm}$ ). This shows the effectiveness of the tumor mimics and ultrasound imaging for guiding HIFU treatments, since the predetermined distance was $10 \mathrm{~mm}$ (average difference: 13\%).
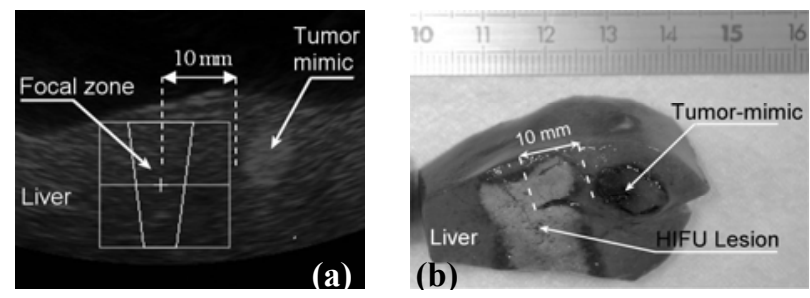

Fig. 6. in vivo HIFU exposure at a distance from a tumor-mimic (a) before exposure on sonogram (b) after HIFU exposure on a sample

In all, 14 HIFU lesions centered on tumor mimics were produced (Fig. 7). The average longitudinal diameter was $23.0 \pm 3.1 \mathrm{~mm}$ (range: $14.0-32.0 \mathrm{~mm}$ ) and the average transversal diameter was $40.3 \pm 6.4 \mathrm{~mm}$ (range: 19.0-58.0 $\mathrm{mm}$ ). The margins around the mimic model were measured in all 3 dimensions; along the axial, orthogonal and longitudinal axes, these measurements were, $14.7 \pm 5.5 \mathrm{~mm}$ (range: $1.0-39.0 \mathrm{~mm}$ ), $5.7 \pm 2.3 \mathrm{~mm}$ (range: $0.0-12.0 \mathrm{~mm}$ ) and $6.4 \pm 3.2 \mathrm{~mm}$ (range: $0.0-18.0 \mathrm{~mm}$ ), respectively. During HIFU exposures, margins of treatment were harder to control along the orthogonal axe using a $2 \mathrm{D}$ imaging system. Overall, the average difference between the predetermined margins of treatment and the actual coagulated margins was $42 \%$.

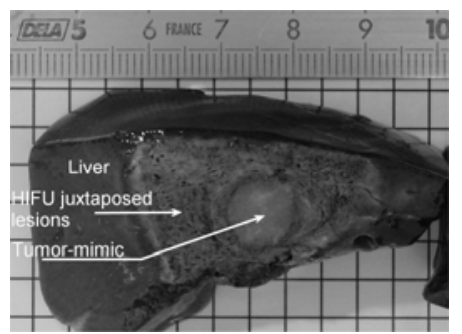

Fig. 7. juxtaposed HIFU exposures into the tumor-mimic

\section{DISCUSSION}

Acoustical parameters in the tumor mimics were close to those in healthy liver tissue [6] and allow the use of this model as a target without inducing any significant modification of ultrasound propagation. The first in vivo study confirmed the feasibility and tolerance of injections. The products used to create the tumor-mimics were inexpensive. The method for embedding the tumor-mimics showed no evidence of complication which could forbid the possibility of transcutaneous injection. However, the aim of this study was to evaluate a specific device used manually and intraoperatively in order to avoid, primarily, the difficulties induced by the ribs. The tumor mimic formed a discrete lesion in hepatic tissue for at least 30 days. The tumor mimics' dimensions were well correlated between sonogram and macroscopic samples. Furthermore, the results of blood analyses performed throughout the study showed that tumor mimics were very well tolerated and therefore could be used as a reference target for evaluating the accuracy of preclinical studies on HIFU treatments without creating complications. The HIFU exposures performed at a distance from the tumor mimic showed that the targeting method was accurate. The differences between the predetermined position of the lesion and the actual location of the coagulated zone did not exceed $13 \%$. However, the lesion volume created during one single exposure was not large enough to treat a 1-cc tumor mimic with margins of $10 \mathrm{~mm}$ in all directions. In liver tumor treatment, this margin was defined to prevent the risk of recurrence after treatment and increase the overall patient survival rate. In this study, it was demonstrated that it was possible to treat a predetermined zone with margins. The right location of the treated regions by HIFU was verify using tumor-mimics. However, the difference between the theoretical margins and the actual margins of treatment were significant. Indeed, some difficulties were encountered in ensuring that the total lesion (composed of multiple exposures) was completely homogeneous around the tumormimics, especially along the axe which is orthogonal to the $2 \mathrm{D}$ ultrasound image. The visualization of HIFU lesions on sonograms has to be improved to ensure that all tissue has been treated.

In conclusion, we have demonstrated that this tumormimic model was well tolerated in the long term (30 days) and has compatible acoustical parameters for evaluating preclinical HIFU treatment in in vivo liver tissue.

\section{ACKNOWLEDGMENT}

The authors wish to thank the staff of the laboratory for experimental surgery for their aid in the animal study.

\section{REFERENCES}

[1] B Cady, RL Jenkins, GD Steele, WD Lewis, MD Stone, WV McDermott, JM Jessup, A Bothe, P Lalor, EJ Lovett, P Lavin, DC Linehan. Ann Surg 1998;227:556-571

[2] JM McLoughlin, EH Jensen, M Malafa, FACS. Resection of Colorectal Liver Metastases: Current Perspectives. Cancer Control 2006 13: 32-41

[3] DJ Scott, WN Young, LM Watamull, GM Lindberg, RV Rege, RJ Brown, DB Jones, "Development of an in-vivo tumour mimic model for learning radiofrequency", J Gastrointest Surg. 2000;4;pp. 620-625

[4] H. Parmentier, D. Melodelima, W.A. N'Djin, J.Y. Chapelon and M. Rivoire "Focal liver ablation by high intensity focused ultrasound: Study on the pig", Proceedings of the $31^{\text {st }}$ Journées de la Société Française de gastroenterology, Lyon, France 2007 in press.

[5] Segal LA, O'Brien WD, "Frequency dependent ultrasonic attenuation coefficient assessment in fresh tissue, IEEE 1983 Ultrasonics Symp Proc, 797-799

[6] GD Ludwig et al., "The velocity of sound through tissues and the acoustical impedance of tissues", J Acoust Soc Am, 1950; 22: 862866. 